CONGENITAL HEART DISEASE

\title{
Improved early ventricular performance with a right ventricle to pulmonary artery conduit in stage 1 palliation for hypoplastic left heart syndrome: evidence from strain Doppler echocardiography
}

\author{
M L Hughes, L S Shekerdemian, C P Brizard, D J Penny
}

Heart 2004;90:191-194. doi: 10.1136/hrt.2003.016675

See end of article for authors' affiliations

Correspondence to: Dr M L Hughes, Cardiology Department, Royal Children's Hospital, Flemington Road, Parkville, Melbourne, Victoria 3052, Australia; marina.hughes@rch. org.au

Accepted 31 July 2003

\begin{abstract}
Objective: To quantify non-invasively right ventricular (RV) performance in infants after stage 1 palliation for hypoplastic left heart syndrome (HLHS).

Design: Prospective, observational study with two dimensional and strain Doppler echocardiography. Setting: Single tertiary paediatric cardiology centre.

Patients: Convenience sample of nine consecutive infants with HLHS. Four whose surgery involved a systemic to pulmonary artery (S-PA) shunt were compared with five whose surgery incorporated a right ventricle to pulmonary artery (RV-PA) conduit.

Methods: Basal RV free wall longitudinal strain rate, systolic strain $(\epsilon)$, and RV percentage area change were calculated during a single assessment between 27-50 days after surgery.

Results: Cardiopulmonary bypass time was longer in patients who underwent RV-PA (226 (30) minutes v 181 (18) minutes, $p=0.03$ ), but cross clamp time, duration of ventilation, and inotrope use did not differ. Two patients in the S-PA group died, on days 29 and 60 after surgery. Peak systolic strain rate $(-1.24$ $(0.19) / s \vee-0.91(0.21) / s, p=0.048)$, peak $\in(-17.8(1.8) \% v-13.4(2.0) \%, p=0.01)$, and RV percentage area change $(56(6) \%$ v $25(6) \%, p<0.01)$ were all greater among RV-PA patients. These indices also tended to be greater in survivors as a group. Ventricular loading conditions (oxygen saturations, diuretic treatment, and blood pressure) were similar in both groups.

Conclusion: Strain Doppler echocardiography shows improved RV longitudinal systolic contractility in patients during convalescence after the RV-PA modification of stage 1 palliation for HLHS compared with those with an S-PA shunt.
\end{abstract}

$\mathrm{S}$ ince Norwood described success with stage 1 surgical palliation (SIP) for hypoplastic left heart syndrome (HLHS), a systemic to pulmonary artery (S-PA), or Blalock-Taussig, shunt has been incorporated to provide pulmonary blood flow. ${ }^{1}$ This surgery results in a precarious balance between systemic and pulmonary blood flow and combines the insults of significant volume loading of the right ventricle (RV) with low diastolic coronary perfusion pressure, ${ }^{2}$ often negatively influencing the postoperative outcome.

A recently re-established modification of S1P incorporates a right ventricle to pulmonary artery (RV-PA) conduit rather than an S-PA shunt. ${ }^{3}$ The goal of this modification is to eliminate aortic diastolic runoff into the pulmonary circulation, thus improving coronary perfusion and ventricular function. While there are limited data to suggest that hospital survival is improved for these patients, ${ }^{4}$ it is unknown whether or not this modification results in improved performance of the RV compared with the traditional approach.

Strain Doppler echocardiography (SDE) provides a noninvasive means of quantifying myocardial deformation. ${ }^{56}$ In principle, systolic strain $(\epsilon)$ reflects the extent of myocardial fibre shortening, while strain rate (SR) reflects the velocity of myocardial shortening. The two indices provide complementary information about segmental myocardial function, are applicable to the RV, and can be measured at the bedside.

This is the first report of the use of SDE techniques to assess RV performance in a cohort of infants during the convalescent phase after SIP for HLHS. The study period, between July 2002 and December 2002, coincided with a planned change in the surgical technique at the Royal Children's Hospital in Melbourne, Australia, enabling direct comparison between infants undergoing the traditional (S-PA shunt) procedure and those undergoing the modified procedure incorporating an RV-PA conduit.

\section{METHODS}

\section{Medical and surgical management}

All neonates studied had typical HLHS, with a small left ventricle, intact ventricular septum, normally related great arteries, and either atresia or critical stenosis of the aortic valve. Ductal patency was maintained with intravenous prostaglandin El. Surgery was undertaken after physiological stability had been achieved, between 2-11 days of age. The surgery was completed with total body perfusion at $24^{\circ} \mathrm{C}$ and maintenance of perfusion through the descending thoracic aorta and right carotid artery during the arch reconstruction. The surgical technique for all patients included construction of a Damus-Kaye-Stansel anastomosis, reconstruction of the aortic arch with treated pericardial patch and atrial septectomy. With standard S1P, pulmonary blood flow was provided through a 3 or $3.5 \mathrm{~mm}$ expanded polytetrafluoroethylene

Abbreviations: $\epsilon$, systolic strain; HLHS, hypoplastic left heart syndrome; $\mathrm{RV}$, right ventricle; $\mathrm{RV} \% \mathrm{~A}-\mathrm{Ch}$, right ventricular percentage area change; $\mathrm{RV}-\mathrm{PA}$, right ventricle to pulmonary artery; S-PA, systemic to pulmonary artery; SIP, stage 1 surgical palliation; SDE, strain Doppler echocardiography; SR, strain rate 
Table 1 Demographic and surgical characteristics of each patient listed in chronological order of surgery

\begin{tabular}{|c|c|c|c|c|c|c|c|c|c|c|c|}
\hline Patient & Anatomy & $\begin{array}{l}\text { Asc Ao } \\
(\mathrm{mm})\end{array}$ & Sex & $\begin{array}{l}\text { Gestation } \\
\text { (weeks) }\end{array}$ & Bthwt (kg) & $\begin{array}{l}\text { Prenatal } \\
\text { diagnosis }\end{array}$ & $\begin{array}{l}\text { Age at } \\
\text { surgery } \\
\text { (days) }\end{array}$ & $\begin{array}{l}\text { CPB time } \\
\text { (min) }\end{array}$ & $\begin{array}{l}\text { Cross clamp } \\
\text { (min) }\end{array}$ & $\begin{array}{l}\text { Ventilated } \\
\text { (h) }\end{array}$ & $\begin{array}{l}\text { Inotrope } \\
\text { support (days) }\end{array}$ \\
\hline \multicolumn{12}{|l|}{ S-PA shunt } \\
\hline 1 & AS/MS & 3 & $M$ & 38 & 3.8 & Yes & 5 & 162 & 57 & 96 & 6 \\
\hline 2 & $\mathrm{AA} / \mathrm{MS}$ & 3 & $M$ & 41 & 3.5 & Yes & 2 & 181 & 64 & 102 & 6 \\
\hline 3 & AS/MA & 5 & $\mathrm{~F}$ & 36 & 2.3 & Yes & 8 & 206 & 43 & 720 & 37 \\
\hline 4 & $\mathrm{AA} / \mathrm{MS}$ & 2 & $M$ & 38 & 3.3 & Yes & 5 & 176 & 55 & 192 & 28 \\
\hline Mean (SD) & & $3.3(1.3)$ & & $38.3(2.1)$ & $3.2(0.7)$ & & $5(2.4)$ & $181(18.3)$ & $55(9)$ & $276(299)$ & 19.3 (15.7) \\
\hline \multicolumn{12}{|c|}{ RV-PA conduit } \\
\hline 5 & $\mathrm{AA} / \mathrm{MS}$ & 2 & $\mathrm{~F}$ & 39 & 2.8 & Yes & 5 & 228 & 80 & 566 & 29 \\
\hline 6 & $\mathrm{AA} / \mathrm{MS}$ & 3 & $M$ & 40 & 4.1 & No & 11 & 216 & 52 & 90 & 8 \\
\hline 7 & $\mathrm{AS} / \mathrm{MS}$ & 6 & $\mathrm{~F}$ & 38 & 3.0 & Yes & 4 & 182 & 44 & 120 & 21 \\
\hline 8 & $\mathrm{AA} / \mathrm{MS}$ & 3 & $M$ & 35 & 2.4 & No & 9 & 259 & 52 & 816 & 33 \\
\hline 9 & $\mathrm{AA} / \mathrm{MS}$ & 2 & $M$ & 38 & 3.2 & Yes & 5 & 249 & 65 & 184 & 13 \\
\hline Mean (SD) & & $3.2(1.6)$ & & 38.0 (1.9) & $3.1(0.6)$ & & $6.8(3)$ & $226(30.2)$ & 59 (14) & 355 (321) & $20.8(10.5)$ \\
\hline $\mathrm{p}$ Value & & 0.96 & & 0.9 & 0.7 & & 0.4 & 0.03 & 0.6 & 0.7 & 0.9 \\
\hline
\end{tabular}

AA, aortic atresia; AS, aortic stenosis; Asc Ao, ascending aorta diameter; Bthwt, birth weight; CPB, cardiopulmonary bypass; $F$, female; $M$, male; $M A$, mitral atresia; MS, mitral stenosis; RV-PA, right ventricle to pulmonary artery; S-PA, systemic to pulmonary artery.

tube inserted between the right subclavian artery and the right pulmonary artery. With the modified SIP a 5 or $6 \mathrm{~mm}$ non-valved expanded polytetrafluoroethylene conduit, constructed between the anterior RV free wall and the distal main pulmonary artery, provided pulmonary flow. The sternum was routinely left open and was closed between the second and seventh postoperative day. In all patients, mixed venous oxygen saturations and arterial blood gas measurements guided strategies to optimise systemic blood flow with ventilation, inotropes, and systemic vasodilators. Infants were managed in the intensive care unit until they were stable off mechanical ventilation. According to practice in our hospital, all patients remained inpatients until the stage 2 procedure with formation of a bidirectional cavopulmonary shunt.

\section{Ultrasound technique}

One sonographer performed all the imaging between the 27th and 50th postoperative day with a Sonos 5500 cardiac ultrasound machine (Philips Medical Systems, Andover, Massachusetts, USA) and simultaneous single lead electrocardiographic monitoring. Patients were imaged on the cardiology ward during quiet rest or sleep. The apical four chamber view was used to calculate the RV percentage area change (RV\%A-Ch) by manually tracing and averaging the difference in area of the RV chamber in systole and diastole over three cardiac cycles. ${ }^{7}$ The RV free wall was then imaged, keeping the longitudinal motion of the wall aligned as near as possible with the ultrasound beam and using the narrowest sector incorporating the myocardium. The velocity scale was adjusted to avoid the aliasing of colour Doppler data and gains were minimised to achieve only myocardial colour saturation. Colour SDE loops, imaging 3-6 consecutive cardiac cycles, were recorded and stored in digital format. The (mean (SD)) frame rate achieved by these methods was 171 (25) frames/s.

\section{Offline analysis}

The SDE loops were analysed offline with dedicated software (Advanced Medical Imaging Development, Rome, Italy). The standardised computational area or region of interest was an 18-20 mm long segment of the RV free wall, measured at end systole in the longitudinal direction from the tricuspid valve annulus towards the apex. The region of interest was orthogonally sliced. The velocity was averaged across the myocardial wall in each slice and SR was integrated over time to derive the natural strain profile, with end diastole as the reference point. ${ }^{58}$ Long axis strain is described by the terms "shortening" in systole (negative strain value) and "lengthening" in diastole (curve returning to baseline point). The timing of end systole and end diastole was derived from an ECG trace and the myocardial velocity profile and values were averaged over 3-6 cardiac cycles. MH repeated the computational analysis of peak systolic SR and $\epsilon$ for all

Table 2 Clinical and echocardiographic characteristics of each patient listed in chronological order of surgery

\begin{tabular}{|c|c|c|c|c|c|c|c|c|c|}
\hline Patient & $\begin{array}{l}\text { Echo post-op } \\
\text { (days) }\end{array}$ & $\begin{array}{l}\text { Heart rate } \\
\text { (beats/min) }\end{array}$ & $\begin{array}{l}\mathrm{SaO}_{2} \\
(\%)\end{array}$ & $\begin{array}{l}\text { Inotrope } \\
(\mu \mathrm{g} / \mathrm{kg} / \mathrm{min})\end{array}$ & TR & $\begin{array}{l}\text { RV \%A-Ch } \\
\text { (\%) }\end{array}$ & $\begin{array}{l}\text { Peak systolic SR } \\
(1 / s)\end{array}$ & $\begin{array}{l}\text { Peak systolic } \epsilon \\
\text { (\%) }\end{array}$ & Outcome \\
\hline \multicolumn{10}{|l|}{ S-PA shunt } \\
\hline 1 & 50 & 149 & 80 & None & Mod & 29 & -1.11 & -16.2 & A \\
\hline 2 & 47 & 167 & 79 & None & Mod & 25 & -0.69 & -12.2 & A \\
\hline 3 & 41 & 136 & 80 & None & Mild & 29 & -0.77 & -11.9 & D (60 days) \\
\hline 4 & 28 & 177 & 82 & Dobutamine 5 & Mild & 17 & -1.08 & -13.4 & D (29 days) \\
\hline Mean (SD) & $41.5(3.4)$ & $157(18)$ & $80(1)$ & & & $25(6)$ & $-0.91(0.21)$ & $-13.4(2.0)$ & \\
\hline \multicolumn{10}{|l|}{ RV-PA conduit } \\
\hline 5 & 34 & 147 & 85 & None & Mild & 49 & -1.54 & -20.4 & A \\
\hline 6 & 27 & 138 & 88 & None & Mod & 62 & -1.06 & -16.5 & A \\
\hline 7 & 36 & 154 & 86 & None & Mild & 55 & -1.18 & -16.7 & A \\
\hline 8 & 31 & 170 & 85 & Dobutamine 2.5 & Mild & 53 & -1.29 & -16.5 & A \\
\hline 9 & 32 & 133 & 82 & None & Mild & 62 & -1.11 & -19.0 & A \\
\hline Mean (SD) & $32(9.7)$ & $148(15)$ & $85(2)$ & & & $56(6)$ & $-1.24(0.19)$ & $-17.8(1.8)$ & \\
\hline $\mathrm{p}$ Value & 0.07 & 0.4 & $<0.01$ & & & $<0.01$ & 0.048 & 0.01 & \\
\hline
\end{tabular}

$\epsilon$, systolic strain; A, alive; D, died; mod, moderate; $\mathrm{RV} \% \mathrm{~A}-\mathrm{Ch}$, right ventricle percentage area change; $\mathrm{SaO}_{2}$, percutaneous oxygen saturation; $\mathrm{SR}$, strain rate; $T R$, tricuspid valve regurgitation. 
patients twice, with a two week interval between analyses. Intraobserver variability was calculated by comparing the values obtained at the first and second analysis. An independent observer (LS) who was blinded to the surgical procedure performed on each patient also computed the peak systolic SR and $\epsilon$ for all patients to estimate interobserver variability. Values of $\mathrm{SR}$ and $\epsilon$ recorded by the blinded observer (LS) at the first reading were used for statistical analysis and comparison between groups.

\section{Statistical analysis}

Data were analysed by Stata Statistical Software, release 7 (Stata Corporation, College Station, Texas, USA). Data reproducibility was expressed both as $95 \%$ limits of agreement $^{9}$ and as Lin's concordance correlation coefficient. ${ }^{10}$ Group values are presented as mean (SD). Continuous data were compared with the $t$ test for independent samples. A probability value of $\mathrm{p}<0.05$ was considered significant.

\section{RESULTS}

Nine infants were studied. The first four underwent standard SIP with an S-PA shunt and the latter five the RV-PA modification. Tables 1 and 2, and fig 1 , present the data for each patient. No patient died in the immediate postoperative period but at the time of writing two patients in the S-PA group had subsequently died, both after sudden collapse.

The duration of cardiopulmonary bypass time was longer in the RV-PA group. One patient in each group (patients 4 and 8) was given low dose inotropic support at the time of the SDE study. The arterial saturation by pulse oximetry was marginally higher in the RV-PA group, though the heart rates of patients were similar in both groups. All patients were receiving diuretic and angiotensin converting enzyme inhibitor. The systolic blood pressure at the time of ultrasound was similar in both groups. No patient had echocardiographic or clinical evidence of recoarctation of the aorta.

The peak systolic SR, peak $\epsilon$, and RV\%A-Ch were all greater in the RV-PA group (table 2). Fig 1 shows the minimum overlap of peak systolic SR and $\epsilon$ values between the two groups. There also tended to be a higher peak $\epsilon$ among survivors $(-16.8(2.6) \%$ for survivors $v-12.6(1.1) \%$ for nonsurvivors, $\mathrm{p}=0.07)$.

\section{Reproducibility}

Lin's $^{10}$ concordance correlation coefficient for intraobserver variability of $\epsilon$ measurements was $0.96 \quad$ (95\% limits of

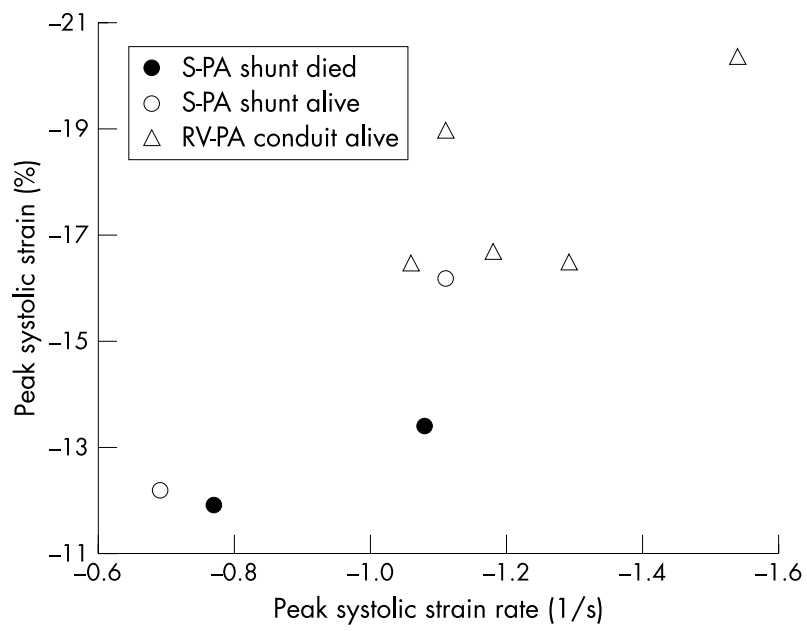

Figure 1 Scatter plot of peak systolic strain $(\epsilon)$ versus peak systolic strain rate (SR) for all patients. RV-PA, right ventricle to pulmonary artery; S-PA, systemic to pulmonary artery. agreement $-1.9,1.4)$, indicating no systematic bias in the separate computational analyses. Lin's concordance correlation coefficient for the interobserver variability of $\epsilon$ measurements was 0.84 (95\% limits of agreement -3.5, 3.6). The reproducibility of SR measurements was similarly accurate.

\section{DISCUSSION}

This study is the first to quantify RV performance noninvasively with SDE and the first to compare two surgical strategies during the convalescent phase after SIP for HLHS. We obtained improved myocardial systolic performance, as shown by a greater magnitude of longitudinal peak systolic SR and $\epsilon$, among patients undergoing the RV-PA modification of SIP. This difference occurred despite the inclusion in this group of two patients who collapsed before postnatal diagnosis

RV function is reduced after standard SIP in infants with HLHS ${ }^{11}$ and contributes to the early death of many patients. In addition to intraoperative myocardial injury, other factors contribute to the poor function. Obligatory volume loading and increased wall stress, accompanied by decreased diastolic coronary perfusion, threaten the balance between myocardial oxygen demand and supply. ${ }^{2}$ With an RV-PA conduit rather than an S-PA shunt, diastolic runoff into the pulmonary circulation is eliminated. Albeit at the expense of a right ventriculotomy, improved coronary perfusion may manifest as improved myocardial systolic function among these patients.

SDE has been validated in experimental work with sonomicrometry ${ }^{5}$ and in adult patients with magnetic resonance tagging ${ }^{6}$ but has only recently been applied clinically to paediatric patients. In principle, $\epsilon$ reflects the extent of myocardial fibre shortening. It parallels changes in ejection performance or stroke volume and is affected by changes in heart rate, both preload and afterload. In our study peak systolic $\epsilon$ paralleled the two dimensional surrogate measure of ejection fraction (RV\%A-Ch). SR reflects the velocity of myocardial shortening and approximates regional contractile function. ${ }^{6}$

\section{Study limitations}

There are a number of limitations to this study. The number of patients is small, and the study is not randomised. Although we included all consecutive infants after the implementation of SDE in our unit and the study period incorporated a predetermined change in surgical practice, further patients should be studied to make recommendations based on our current findings. However, the theoretical physiological advantages of an RV-PA conduit, coupled with a widespread anecdotal perception of improved perioperative stability in these patients, have shifted the surgical approach in a number of international centres including our own. It is unlikely that a study with true randomisation of the surgical procedure will be performed in the near future.

It can be argued that the greater $\epsilon$ or systolic deformation in the RV-PA group was the result of a more volume loaded RV. However, there is no clear haemodynamic reason for this. The RV-PA conduit is restrictive, with predominantly forward flow into a low resistance pulmonary circulation. Oxygen saturations were clinically similar in both groups. All patients were receiving diuretics and afterload reduction treatment in the form of an angiotensin converting enzyme inhibitor.

We have only reported measures of systolic function. Diastolic SR indices were measured but extremely high SR values for the S-PA patients resulted in aliasing and prevented accurate quantification of diastolic SR data. Computational techniques are being developed to dephase these data. 
The study is cross sectional, with single measurements reported during the convalescent phase four to seven weeks after surgery. Longitudinal studies are in progress to evaluate these patients over time.

\section{Conclusion}

We have shown that SDE can provide a non-invasive means of quantifying the RV function of infants in a clinical setting with the use of commercially available equipment. Patients during convalescence after the RV-PA modification of SIP for HLHS appear to have greater RV longitudinal systolic contractility than do those with an S-PA shunt.

\section{ACKNOWLEDGEMENT}

Marina Hughes is supported by a KPMG Foundation Fellowship in Cardiovascular Disease Research.

\section{Authors' affiliations}

M L Hughes, D J Penny, Department of Cardiology, Royal Children's Hospital, Melbourne, Australia

L S Shekerdemian, Department of Intensive Care, Royal Children's Hospital, Melbourne

C P Brizard, Department of Cardiac Surgery, Royal Children's Hospital, Melbourne

\section{REFERENCES}

1 Norwood WI, Kirklin JK, Sanders SP. Hypoplastic left heart syndrome: experience with palliative surgery. Am J Cardiol 1980;45:87-91.

2 Donnelly JP, Raffel DM, Shulkin BL, et al. Resting coronary flow and coronary flow reserve in human infants after repair or palliation of congenital heart defects as measured by positron emission tomography. J Thorac Cardiovasc Surg 1998;115:103-10.

3 Kishimoto $\mathrm{H}$, Kawahira $\mathrm{Y}$, Kawata $\mathrm{H}$, et al. The modified Norwood procedure on a beating heart. J Thoracic Cardiovasc Surg 1999; 1 18:1130-2.

4 Pizarro C, Malec E, O Maher K, et al. Right ventricle to pulmonary artery conduit improves outcome after Norwood procedure for hypoplastic left heart syndrome [abstract]. Circulation 2002;106(suppl II):1961.

5 Urheim S, Edvardsen T, Torp T, et al. Myocardial strain by Doppler echocardiography: validation of a new method to quantify regional myocardial function. Circulation 2000;102:1158-64.

6 Edvardsen T, Gerber BL, Garot J, et al. Quantitative assessment of intrinsic regional myocardial deformation by Doppler strain rate echocardiography in humans: validation against three-dimensional tagged magnetic resonance imaging. Circulation 2002;106:50-6

7 Altman K, Printz BF, Solowiejczyk DE, et al. Two-dimensional echocardiographic assessment of right ventricular function as a predictor of outcome in hypoplastic left heart syndrome. Am J Cardiol 2000;86:964-8.

8 D'hooge J, Heimdal A, Jamal F, et al. Regional strain and strain rate measurements by cardiac ultrasound: principles, implementation and limitations. Eur J Echocardiogr 2000;1:154-70.

9 Bland J, Altman DG. Statistical methods for assessing agreement between two methods of clinical measurement. Lancet 1986;i:307-10.

10 Lin LIK. A concordance correlation coefficient to evaluate reproducibility. Biometrics 1989:45:255-68.

11 Kimball TR, Witt SA, Khoury PR, et al. Automated echocardiographic analysis of systemic ventricular performance in hypoplastic left heart syndrome. J Am Soc Echocardiogr 1996;9:629-36.

\section{IMAGES IN CARDIOLOGY}

\section{Acute aortic dissection induced renovascular hypertension}

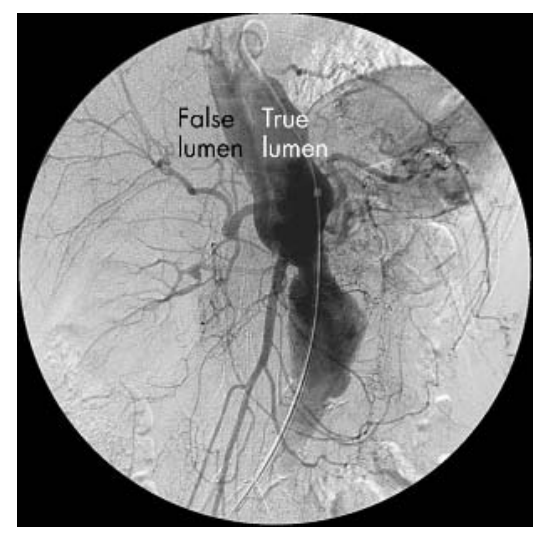

$\Delta$ lthough percutaneous renal angioplasty has been extensively used for fibromuscular or atherosclerotic renovascular hypertension, few cases have been reported in patients with aortic dissection. We describe stent angioplasty for severe stenosis of the right renal artery in a patient with a Stanford type B aortic dissection.

A 71 year old woman, who had suffered from hypertension for 30 years, consulted our hospital because of an acute onset of sharp back pain. Computed tomography showed a persistent false lumen originating from the descending thoracic aorta with termination at the right renal artery, indicating an acute aortic dissection, Stanford type B. After hospitalisation her blood pressure was difficult to control such that her systolic blood pressure frequently exceeded $180 \mathrm{~mm} \mathrm{Hg}$, in spite of the intravenous administration of nicardipine. Additionally, her serum creatinine developed from $8 \mathrm{~g} / \mathrm{m}^{3}$ up to $31 \mathrm{~g} / \mathrm{m}^{3}$, and her renin activity was elevated from $0.428 \mu \mathrm{g} / \mathrm{m}^{3} / \mathrm{s}$ up to $2.636 \mu \mathrm{g} / \mathrm{m}^{3} / \mathrm{s}$, within 2 weeks. From these observations, we suggested that the patient's hypertension was caused by stenosis of the renal artery induced by aortic dissection. Digital subtraction angiography (upper panel) and percutaneous renal angiography (lower panel) confirmed stenosis of the right renal artery, which was compressed by the false aortic channel. Therefore, percutaneous renal stent angioplasty was performed on the right renal artery. After stenting, the patient's blood pressure returned to baseline.

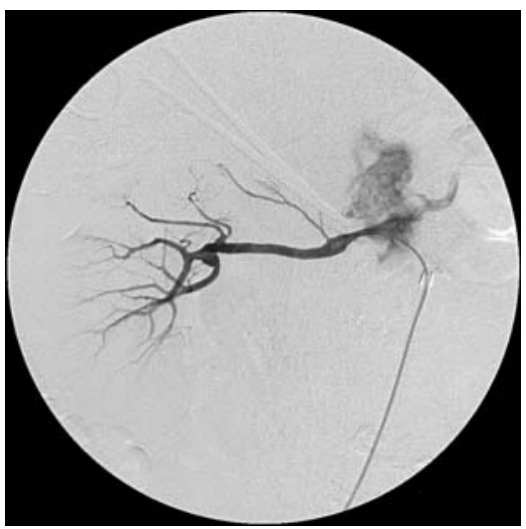

M Shimoyama

G Igawa

M Hashimoto

masaki-tky@umin.umin.ac.jp 